\title{
Resultados a medio-largo plazo del tratamiento monopuerto SuPerLap de la hernia inguinal
}

\author{
R.A. Hernández-Rodríguez, M.J. Rosell Echevarría, F.D. Ravelo Díaz, V. Villamil, E.L. Pérez Etchepare Figueroa \\ Servicio de Cirugía Pediátrica. Hospital Universitario Nuestra Señora de Candelaria. Santa Cruz de Tenerife
}

\section{RESUMEN}

Objetivos. La laparoscopia en el tratamiento de la hernia inguinal está cada vez más presente en muchos hospitales. El uso de ópticas con canal de trabajo no está tan extendido. Se presenta la experiencia a largo plazo en la aplicación de la técnica SuPerLap (sutura percutánea laparoasistida) propuesta por Rosell y cols.(1) para la reparación de hernias epigástricas en el tratamiento monopuerto, percutáneo de las hernias inguinales mediante el uso de ópticas con canal de trabajo.

Material y método. Serie quirúrgica de hernia inguinal congénita en varones (febrero de 2017-diciembre de 2020). Se utilizó: pleuroscopio de $5 \mathrm{~mm}-0^{\circ}$ con canal de trabajo de $3,5 \mathrm{~mm}$; aguja epidural $20 \mathrm{G}$; suturas de polipropileno y poliéster 3/0; disector Maryland laparoscópico (36 $\mathrm{cm}-3,5 \mathrm{~mm}$ ).

Resultados. Se realizaron 384 herniorrafias inguinales según técnica SuPerLap en 295 varones (206 unilaterales, 89 bilaterales). En 24 casos bilaterales $(26,95 \%)$ el diagnóstico preoperatorio fue unilateral. La edad media fue de dos años ( 2 semanas-13 años). El tiempo medio quirúrgico fue 14 minutos (6-50 min) en unilaterales, 27 (14-80 min) en bilaterales. Hubo dos casos de lesión de vasos epigástricos y una recidiva precoz en un neonato, reintervenido satisfactoriamente mediante técnica SuPerLap. En un seguimiento de 1-36 meses no hubo complicaciones tardías.

Conclusiones. El uso de ópticas con canal de trabajo según técnica SuPerLap posibilita prescindir de puertos adicionales en el tratamiento de la hernia inguinal. Permite resultados funcionales comparables y cirugía sin cicatrices visibles. Minimiza la manipulación del cordón espermático. La laparoscopia permite el tratamiento concomitante de defectos no diagnosticados previamente.

Palabras Clave: Hernia inguinal; Herniorrafia; Laparoscopia; Monopuerto; Toracoscopia; Cirugía sin cicatrices.

\section{Mid- TO LONG-TERM RESUlTS OF SUPERLAP SINGLE-PORT TREATMENT IN INGUINAL HERNIA}

\begin{abstract}
Introduction. Laparoscopic treatment of inguinal hernia is gaining popularity in many hospitals, but the use of working channel scopes is not as widely extended. We present our long-term experience with the SuPerLap (laparoscopic-assisted percutaneous suture) technique described by Rosell et al.(1) for epigastric hernia repair in the percutaneous, single-port treatment of inguinal hernia using working channel scopes.

Materials and methods. A retrospective analysis of a series of male patients with congenital inguinal hernia undergoing surgery from February 2017 to December 2020 was carried out. A $5 \mathrm{~mm}-0^{\circ}$ pleuroscope with a $3.5 \mathrm{~mm}$ working channel, a $20 \mathrm{G}$ epidural needle, a 36 $\mathrm{cm} / 3.5 \mathrm{~mm}$ laparoscopic Maryland dissector, and 3-0 polypropylene and polyester sutures were used.
\end{abstract}

Results. 384 inguinal hernia repairs using the SuPerLap technique were performed in 295 male patients - 206 unilateral repairs and 89 bilateral repairs. In 24 bilateral cases (26.95\%), preoperative diagnosis had been unilateral. Mean age was two years ( 2 weeks-13 years). Mean operating time was 14 minutes $(6-50 \mathrm{~min})$ for unilateral repair, and 27 minutes (14-80 min) for bilateral repair. There were two cases of epigastric vessel damage, and one case of early recurrence in a newborn, who successfully underwent re-intervention using the SuPerLap technique. No late complications were recorded after a mean follow-up of 1-36 months.

Conclusions. Working channel scopes using the SuPerLap technique avoid additional ports in inguinal hernia repair. They allow for excellent functional results, without visible scars, and minimize spermatic cord manipulation. Laparoscopy allows previously undiagnosed defects to be concomitantly treated.

Key Words: Inguinal hernia; Hernia repair; Laparoscopy; Single port; Thoracoscope; Scarless surgery.

\section{OBJETIVOS}

La hernia inguinal es uno de los diagnósticos más frecuentes por los que consulta el paciente pediátrico. El tratamiento laparoscópico ha ganado terreno al tradicional por cirugía abierta progresivamente en muchos hospitales, debido fundamentalmente a la mínima manipulación del cordón es-
Correspondencia: Dra. Raquel Angélica Hernández-Rodríguez.

E-mail address: raquelhache@hotmail.com 


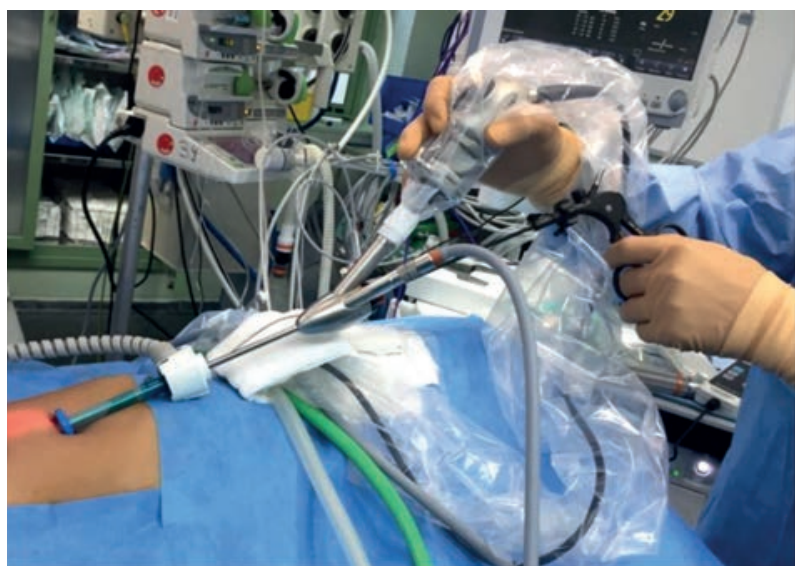

Figura 1. Óptica de $5 \mathrm{~mm}$ con canal de trabajo de $3,5 \mathrm{~mm}$ a través del cual se introduce un disector laparoscópico tipo Maryland de $36 \mathrm{~cm}$.

permático en el varón, a los superiores resultados estéticos y a una recuperación $\operatorname{precoz}^{(2,3)}$.

La técnica monopuerto descrita por Patkowski y cols. ${ }^{(3)}$ consistente en la sutura percutánea del anillo inguinal profundo bajo visión laparoscópica ha contribuido a la mejora de las ventajas aportadas por la laparoscopia, pero se ve limitada por una curva de aprendizaje larga y a dificultades técnicas. Por otro lado, el uso propuesto por Rosell y cols.(1) para ópticas con canal de trabajo en el tratamiento laparoscópico monopuerto de hernias epigástricas mediante técnica SuPerLap demuestra las ventajas de implementar su uso en técnicas reconstructivas de la pared abdominal. Se presenta una modificación del tratamiento laparoscópico percutáneo de la hernia inguinal mediante técnica SuPerLap (sutura percutánea laparoasistida) con utilización de una óptica con canal de trabajo, y nuestros resultados a medio y largo plazo.

\section{MATERIAL Y MÉTODOS}

Análisis descriptivo retrospectivo de una serie de pacientes varones con diagnóstico de hernia inguinal indirecta intervenidos mediante reparación laparoasistida por técnica SuPerLap en nuestro centro entre febrero de 2017 y diciembre de 2020.

Se excluyó a pacientes con historia de cirugía abdominal previa y a pacientes con diagnóstico de hernia inguinal complicada que requirió cirugía urgente.

\section{Descripción de la técnica SuPerLap}

Mediante puerto único transumbilical, se mantiene neumoperitoneo a $8-10 \mathrm{mmHg}$ y se introduce una óptica de $5 \mathrm{~mm}-0^{\circ}$ con canal de trabajo de $3,5 \mathrm{~mm}$. Se localiza el anillo inguinal profundo externamente, presionando con un mosquito o similar bajo visualización laparoscópica para ubicar el lugar de punción.

Para la sutura percutánea se utiliza una aguja epidural de $20 \mathrm{G}$ a través de la cual se pasa un hilo de polipropileno de

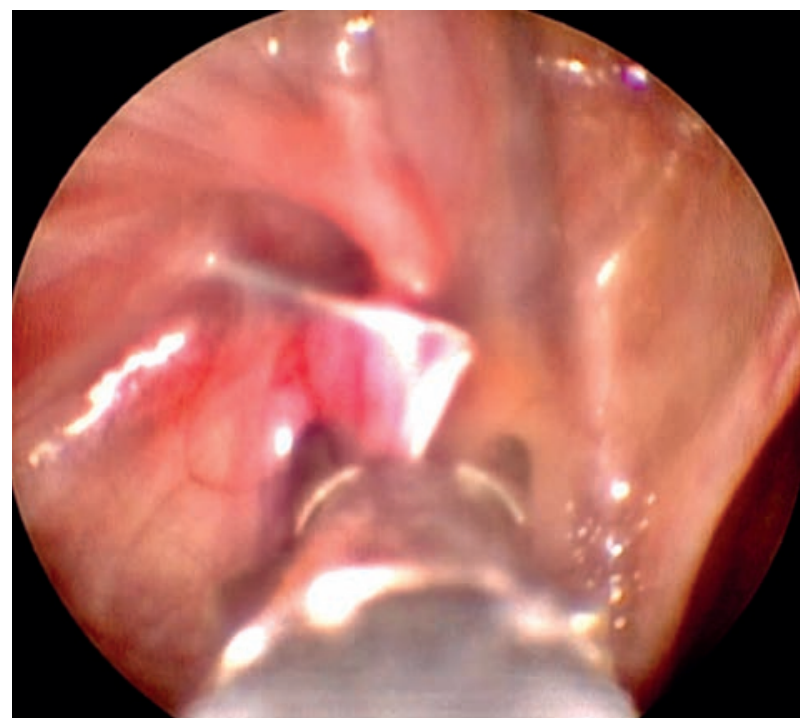

Figura 2. Visión de la sutura de la hemicircunferencia lateral con ayuda del disector auxiliar.

$3 / 0$. Se curva parcialmente la punta hasta unos $85-90^{\circ}$, consiguiendo un trayecto perpendicular al eje de la aguja de unos 2 $\mathrm{cm}$. Sujetando la aguja epidural en su base entre el primer y tercer dedo, y estabilizando a nivel del ángulo con el segundo dedo, se realiza una punción perpendicular en el lugar previamente localizado con el mosquito, hasta atravesar la fascia y visualizar en el espacio preperitoneal la punta de la aguja.

Con ayuda de un disector tipo Maryland de $36 \mathrm{~cm}$ de largo y calibre de $3,5 \mathrm{~mm}$, introducido a través del canal de trabajo de la óptica descrita previamente (Fig. 1), se aproxima el peritoneo del anillo inguinal profundo hacia la aguja, realizando en primer lugar la sutura de la hemicircunferencia lateral (Fig. 2).

Se deja un asa larga de esta sutura, llevándola con la pinza hacia el orificio inguinal profundo contralateral, y se retira la aguja con cuidado de preservar la sutura de polipropileno in situ. A continuación se repite el proceso con la hemicircunferencia medial, utilizando una sutura de poliéster 3/0, asegurando que la punción sea en el mismo punto que el paso previo y que el asa de poliéster quede holgada y dentro del asa de polipropileno. Se retira la aguja y se exterioriza la sutura de poliéster al retirar la sutura de polipropileno (Fig. 3). Finalmente se anuda el doble hilo de poliéster mientras se tracciona caudal y medialmente del teste ipsilateral, quedando el nudo en el espacio subcutáneo. Se cierra el acceso umbilical según técnica habitual del cirujano con sutura reabsorbible.

\section{RESULTADOS}

Se realizaron en nuestro centro 384 herniorrafias percutáneas laparoasistidas según técnica SuPerLap en 295 varones: en 206 casos el diagnóstico fue de hernia unilateral y en 89, 


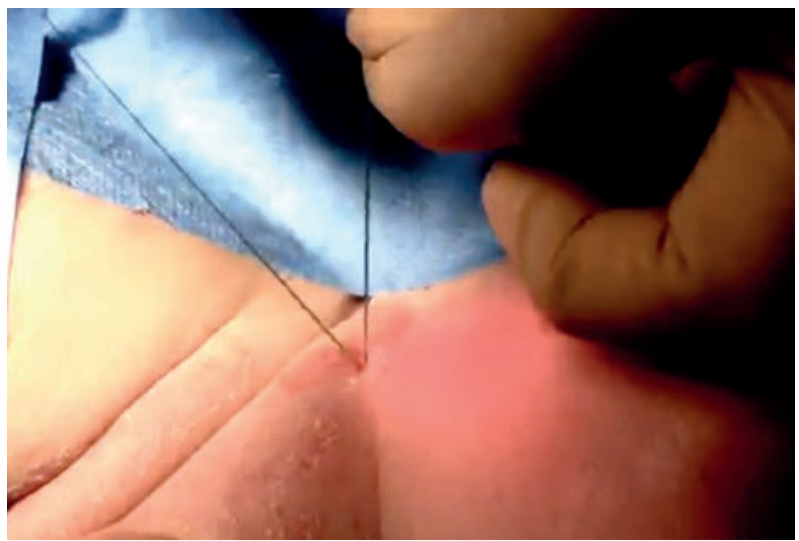

Figura 3. Tras la exteriorización de la sutura de poliéster, se realiza el anudado extracorpóreo. El nudo queda en el subcutáneo, consiguiendo una cirugía de la hernia inguinal sin cicatrices visibles.

de hernia bilateral. En 24 de los casos bilaterales (26,95\%) el diagnóstico preoperatorio fue de hernia unilateral, constatándose durante la laparoscopia una persistencia del conducto peritoneovaginal contralateral; en un paciente el diagnóstico de hernia inguinal bilateral fue realizado durante una piloromiotomía laparoscópica. La edad media a la intervención fue de dos años (2 semanas-13 años). El tiempo medio operatorio en hernias unilaterales fue de 14 minutos (6-50 $\mathrm{min})$, y en bilaterales, de 27 minutos (14-80 $\mathrm{min}$ ).

Como complicaciones intraoperatorias, en dos casos se lesionaron accidentalmente los vasos epigástricos, resolviendo en pocos minutos mediante compresión externa únicamente.

En cuanto a complicaciones postoperatorias inmediatas, hubo una recidiva de la hernia al séptimo día postoperatorio en un neonato de $2.500 \mathrm{~g}$ reintervenido con éxito al día siguiente mediante la misma técnica.

En un seguimiento medio de 1-36 meses no se encontró hidrocele postoperatorio, recidivas u otras complicaciones tardías de la técnica.

\section{DISCUSIÓN}

La reparación tradicional laparoscópica de la hernia inguinal sustituye la necesidad de inguinotomía de la técnica abierta por la colocación de dos o tres puertos para realizar la sutura del orificio inguinal profundo(2).

La técnica monopuerto descrita por Patkowski y cols. ${ }^{(3)}$, consistente en la sutura percutánea del anillo inguinal profundo bajo visión laparoscópica, ha contribuido a la mejora de las ventajas aportadas por la laparoscopia. Se ve limitada por una curva de aprendizaje larga debido a la imposibilidad de triangulación al no utilizar instrumental laparoscópico, por lo que en ocasiones se recurre a un puerto adicional para completar la cirugía de manera segura debido al riesgo de lesión de los vasos ilíacos o espermáticos e incluso el conducto deferente.
Se han descrito otras técnicas como la hidrodisección percutánea bajo visión laparoscópica ${ }^{(4)}$ para incrementar la seguridad de la técnica monopuerto, aunque consideran la adición de puertos adicionales en algunas situaciones especiales. En otros casos, se utiliza una única incisión transumbilical a través de la cual se introducen dos trócares en paralelo(5). Una revisión sistemática comparando 15 series quirúrgicas en laparoscopia tradicional y por puerto único para el tratamiento de la hernia, con una $n=3.680$ pacientes pediátricos, concluyó que la sutura extracorpórea por puerto único fue superior a la sutura intracorpórea tradicional en términos de recidiva de la hernia y tiempo quirúrgico(6).

En el caso de las hernias epigástricas, Correia-Pinto y cols. ${ }^{(7)}$ describió una técnica mínimamente invasiva que evita la incisión en línea media abdominal, a la vez que permite una visualización directa del defecto. Sin embargo, requiere un puerto adicional para realizar la sutura laparoscópica de la misma. Rosell y cols. ${ }^{(1)}$ publicó la técnica SuPerLap para las herniorrafias epigástricas, un principio que modifica la técnica propuesta por Correia-Pinto y cols. ${ }^{(7)}$ y que solventa la necesidad de un puerto adicional mediante el uso de una óptica con canal de trabajo y sutura extracorpórea del defecto, con resultados comparables a los de la técnica tradicional.

El principio de la técnica SuPerLap puede aplicarse a otras intervenciones de tipo resectivo y reconstructivo por igual con una adecuada planificación. En nuestro centro se ha implementado en la reparación de hernia inguinal en el varón según la técnica descrita en este artículo, pero también en la simpatectomía toracoscópica en el tratamiento de las hiperhidrosis primarias y en la cirugía monopuerto ecoguiada de los quistes de duplicación intestinal, siendo de elección en otras técnicas con un alto grado de reproducibilidad.

Como ventaja principal, en la hernia inguinal del varón conseguimos evitar la necesidad de trócares adicionales con la óptica de $5 \mathrm{~mm}$ con canal de trabajo, con resultados comparables a otras técnicas monopuerto. Las ventajas adicionales de la técnica SuPerLap radican principalmente en que el uso del disector facilita la guía para suturar de manera segura el peritoneo del orificio inguinal profundo, evitando la lesión del conducto deferente o los vasos espermáticos, y disminuyendo el tiempo quirúrgico también al evitar el tiempo de colocación de trócares adicionales y, por tanto, el tiempo total de anestesia.

Otra ventaja de la técnica SuPerLap, comparable al abordaje laparoscópico tradicional, es un mejor control del dolor postoperatorio, en algunos casos sin requerimiento alguno de administración de analgesia domiciliaria. Aunque en caso de hernias inguinales complicadas la laparoscopia podría ser de utilidad en la exploración intraabdominal de las estructuras herniadas y de la cavidad peritoneal, por razón del análisis descriptivo de la técnica excluimos de esta serie a pacientes con diagnóstico de hernia inguinal complicada.

Seleccionar adecuadamente el paciente a quien ofrecer la técnica puede ser fundamental, y pasa obligatoriamente por excluir a aquellos que no toleren la laparoscopia. En nuestro 
caso, creemos que la recidiva precoz descrita en nuestra serie podría ser consecuencia de incluir un paciente recién nacido al inicio de nuestra curva de aprendizaje. Por otro lado, el antecedente de cirugía abdominal previa podría ser una contraindicación relativa, y aunque por motivo del análisis descriptivo de esta serie se excluyó a este grupo de pacientes, en nuestra práctica diaria, informando previamente a los padres de las posibilidades de conversión, se procede a ofrecer de primera línea SuPerLap. En el caso de un paciente con antecedentes de enterocolitis necrotizante perforada intervenida en el periodo neonatal con diagnóstico de hernia inguinal a los ocho meses, se abordó inicialmente mediante técnica SuPerLap; debido al hallazgo de adherencias que comprometían la disección de la anatomía del anillo inguinal se decidió convertir a laparoscopia convencional.

La técnica es reproducible de igual manera en niñas, sin el riesgo de lesión de estructuras del cordón espermático que entraña la cirugía en el varón. Sin embargo, preferimos realizar en esta población la cauterización laparoscópica del saco herniario descrita por Godoy-Lenz y cols., conocida como BURNIA $^{(8)}$, dada la inocuidad y la reducción de los tiempos quirúrgicos que supone, con resultados comparables a la cirugía abierta. La cauterización del saco en varones podría ocasionar daño por contigüidad en el conducto deferente y los vasos espermáticos, por lo que preferimos reservar esta técnica para el tratamiento de la hernia inguinal en niñas e indicar la técnica SuPerLap en varones.

De cara a conseguir un adecuado entrenamiento en la cirugía de la hernia inguinal, Moreira-Pinto y cols. ${ }^{(9)}$ propone un modelo realizado a partir de dos guantes quirúrgicos y una óptica, sujeto a modificaciones según la técnica a entrenar. El entrenamiento permite una aceleración de la curva de aprendizaje ${ }^{(10)}$. En nuestro centro ofrecemos de primera línea la técnica SuPerLap para la reparación de la hernia inguinal, con buena aceptación por parte de los padres, y es nuestro tratamiento de elección incluso con cirujanos actualmente en entrenamiento en cirugía endoscópica.

En conclusión, la aplicación del principio SuPerLap en la cirugía laparoscópica monopuerto de la hernia inguinal en niños es factible y reproducible, incluso en cirujanos actualmente en entrenamiento en cirugía endoscópica con una adecuada selección de pacientes al inicio de su curva de aprendizaje. Permite conseguir resultados funcionales y estéticos comparables sin puertos adicionales. Ofrece la ventaja adicional de disminuir el tiempo quirúrgico y anestésico, sobre todo en caso de hernia inguinal bilateral. Minimiza la lesión de los elementos del cordón espermático. Permite la detección de persistencia metacrónica del conducto peritoneovaginal y de otros defectos de pared abdominal y la posibilidad de tratamiento de estos y otros procesos en el mismo acto quirúrgico sin necesidad de trócares adicionales.

\section{BIBLIOGRAFÍA}

1. Rosell Echevarría MJ, Hernández Rodríguez RA, Valverde S, Villamil V, Pérez-Etchepare E. Single port treatment of epigastric hernias using an operating scope. J Ped Endosc Surg [Internet]. 2021; 3: 97-100.

2. Montupet P, Esposito C. Laparoscopic treatment of congenital inguinal hernia in children. J Pediatr Surg. 1999; 34(3): 420-3.

3. Patkowski D, Czernik J, Chrzan R, Jaworski W, Apoznanski W. Percutaneous internal ring suturing: a simple minimally invasive technique for inguinal hernia repair in children. J Laparoendosc Adv Surg Tech A. 2006; 16(5): 513-7.

4. Li S, Li M, Wong KKY, Liu L, Tam PKH. Laparoscopically assisted simple suturing obliteration (LASSO) of the internal ring using an epidural needle: a handy single-port laparoscopic herniorrhaphy in children. J Pediatr Surg. 2014; 49(12): 1818-20.

5. Wang F, Shou T, Zhong H. Is two-port laparoendoscopic single-site surgery (T-LESS) feasible for pediatric hydroceles? Single-center experience with the initial 59 cases. J Pediatr Urol. 2018; 14(1): 67.e1-67.e6.

6. Maat S, Dreuning K, Nordkamp S, van Gemert W, Twisk J, Visschers R, et al. Comparison of intra- and extra-corporeal laparoscopic hernia repair in children: A systematic review and pooled data-analysis. J Pediatr Surg [Internet]. 2021; 56(9): 1647-56.

7. Moreira-Pinto J, Correia-Pinto J. Scarless laparoscopic repair of epigastric hernia in children. Hernia. 2015; 19(4): 623-6.

8. Novotny NM, Puentes MC, Leopold R, Ortega M, Godoy-Lenz J. The Burnia: Laparoscopic sutureless inguinal hernia repair in girls. J Laparoendosc Adv Surg Tech A. 2017; 27(4): 430-3.

9. Coelho A, Mendes L, Correia-Pinto J, Moreira-Pinto J. Preliminary assessment of a dry-lab model for laparoscopic percutaneous inguinal ring suture training. J Laparoendosc Adv Surg Tech A. 2018; 28(9): 1121-4.

10. Barroso C, Etlinger P, Alves AL, Osório A, Carvalho JL, LamasPinheiro R, et al. Learning curves for laparoscopic repair of inguinal hernia and communicating hydrocele in children. Front Pediatr. 2017; 5: 207. 